\title{
Regulation of hippocampal synaptic plasticity thresholds and changes in exploratory and learning behavior in dominant negative NPR-B mutant rats
}

\section{Gleb Barmashenko 1,2, Jens Buttgereit ${ }^{3,4}$, Neil Herring ${ }^{4,5}$, Michael Bader ${ }^{4}$, Cemil Özcelik ${ }^{3,4}$, Denise Manahan-Vaughan ${ }^{2}$ and Karl H. Braunewell ${ }^{1}$ *}

${ }^{1}$ Guest Group, In vitro-Electrophysiology Laboratory, Department of Neurophysiology, Medical Faculty, Ruhr University Bochum, Bochum, Germany

2 Department of Neurophysiology, Medical Faculty, Ruhr University Bochum, Bochum, Germany

${ }^{3}$ Experimental and Clinical Research Center, Max Delbrück Center for Molecular Medicine, Charité Medical Faculty, Berlin, Germany

${ }^{4}$ Max Delbrück Center for Molecular Medicine, Berlin, Germany

${ }^{5}$ Department of Physiology, Anatomy and Genetics, Burdon Sanderson Cardiac Science Centre - BHF Centre of Research Excellence, University of Oxford, Oxford, UK

\section{Edited by:}

Clint Lawrence Makino,

Massachusetts Eye and Ear Infirmary

and Harvard Medical School, USA

\section{Reviewed by:}

Yi-Ping Hsueh, Institute of Molecular

Biology - Academia Sinica, Taiwan

Teresa Duda, Salus University, USA

\section{${ }^{*}$ Correspondence:}

Karl H. Braunewell, Guest Group, In vitro-Electrophysiology Laboratory, Department of Neurophysiology, Medical Faculty, Ruhr University Bochum, Universitätsstraße 150, Building MA 4/150, 44780 Bochum, Germany

e-mail: kbraunewell@gmail.com

${ }^{+}$Present address:

Cemil Özcelik, KLINIKUM VEST GmbH, Dorstener Straße 151, 45657 Recklinghausen, Germany
The second messenger cyclic GMP affects synaptic transmission and modulates synaptic plasticity and certain types of learning and memory processes. The impact of the natriuretic peptide receptor B (NPR-B) and its ligand C-type natriuretic peptide (CNP), one of several cGMP producing signaling systems, on hippocampal synaptic plasticity and learning is, however, less well understood. We have previously shown that the NPR-B ligand CNP increases the magnitude of long-term depression (LTD) in hippocampal area CA1, while reducing the induction of long-term potentiation (LTP). We have extended this line of research to show that bidirectional plasticity is affected in the opposite way in rats expressing a dominant-negative mutant of NPR-B (NSE-NPR-B $\Delta K C$ ) lacking the intracellular guanylyl cyclase domain under control of a promoter for neuron-specific enolase. The brain cells of these transgenic rats express functional dimers of the NPR-B receptor containing the dominant-negative NPR-B $\triangle K C$ mutant, and therefore show decreased CNP-stimulated cGMP-production in brain membranes. The NPR-B transgenic rats display enhanced LTP but reduced LTD in hippocampal slices. When the frequency-dependence of synaptic modification to afferent stimulation in the range of $1-100 \mathrm{~Hz}$ was assessed in transgenic rats, the threshold for both, LTP and LTD induction, was shifted to lower frequencies. In parallel, NPR-B $\triangle K C$ rats exhibited an enhancement in exploratory and learning behavior. These results indicate that bidirectional plasticity and learning and memory mechanism are affected in transgenic rats expressing a dominant-negative mutant of NPR-B. Our data substantiate the hypothesis that NPR-B-dependent cGMP signaling has a modulatory role for synaptic information storage and learning.

Keywords: cGMP, exploratory, hippocampus, LTP, LTD, metaplasticity, NPR-B, memory

\section{INTRODUCTION}

The natriuretic peptides, ANP, BNP, and CNP (A-, B-, and C- type natriuretic peptide) and their receptors, the natriuretic peptide receptors (NPRs) are widely distributed in the central nervous system (CNS). They constitute a peptide hormone-receptor signaling system with a variety of potential roles in modulating physiological brain functions (Potter et al., 2009; Potter, 2011). Whereas ANP and BNP activate the type I transmembrane guanylyl cyclase receptor, natriuretic peptide receptor-A (NPR-A), CNP activates a related cyclase, the natriuretic peptide receptor- $\mathrm{B}$ (NPR-B), leading to the production of the second messenger cGMP (Potter et al., 2009). NPR-A and -B display several functional domains, including an extracellular ligand-binding domain, a transmembrane domain, and an intracellular domain, which consists of a kinase homology domain (KHD), a hinge region, and a guanylyl cyclase domain catalyzing the conversion of Mg-GTP into cGMP. A ligand-dependent receptor homodimerization is essential for the enzymatic activity of the guanylyl cyclase domain (Potter, 2011).

Natriuretic peptide receptor-B leads to various physiological effects ranging from bone growth (Bartels et al., 2004; Potter et al., 2009; Potter, 2011) to CNS effects, such as axonal sprouting in dorsal root ganglion neurons (Schmidt et al., 2007; Potter et al., 2009), effects on synaptic plasticity (Decker et al., 2008, 2009, 2010), and anxiogenic effects in rats and humans (Jahn et al., 2001; Kellner et al., 2003). CNP and its receptor NPR$\mathrm{B}$ are widely expressed in the rat brain including expression in the limbic system, particularly in the hippocampal regions CA1-3 (Langub etal., 1995; Herman etal., 1996). In the hippocampus NPR-B is expressed in pyramidal cells and GAD (65/67)-immunopositive interneurons. Sub-cellular expression of NPR-B can be demonstrated in neuronal process in primary hippocampal cell cultures (Brackmann et al., 2005). The second messenger cGMP and cGMP signaling-cascades are implicated 
in the modulation of long term potentiation (LTP), long-term depression (LTD) as well as other forms of synaptic plasticity, such as short-term plasticity (Schuman and Madison, 1991; Arancio etal., 1996; Son etal., 1998). The effect of cGMP on synaptic plasticity has been attributed to the activity of soluble guanylyl cyclases (sGCs; Arancio et al., 1996). Moreover, downstream cGMP targets such as cyclic nucleotide-gated channels and cGMP-dependent kinases (cGK or protein kinase G, PKG) contribute to different forms of synaptic plasticity (Klyachko et al., 2001; Kleppisch et al., 2003; Liu et al., 2003). More recently, we investigated whether activation of another cGMP-signaling system, the membrane guanylyl cyclase NPR-B, via activation of by its ligand $\mathrm{CNP}$ has an effect on hippocampal synaptic plasticity (Decker et al., 2008, 2009, 2010). When LTD and LTP stimulation was applied in area CA1 at 1 and $5 \mathrm{~Hz}$ and 30 $100 \mathrm{~Hz}$, respectively, CNP increased the magnitude of LTD while LTP induction was reduced. Thus, in the presence of CNP the threshold for LTP induction was shifted to higher stimulus frequencies (Decker et al., 2010). C-type natriuretic peptide (CNP) also decreased hippocampal network oscillations in adult rats in vitro, which are believed to be involved in storage of information and memory consolidation in vivo (Decker et al., 2009). In line with these results earlier work showed that direct application of CNP into the lateral brain ventricles affects the performance of rats in a passive avoidance learning paradigm (Telegdy et al., 1999, 2000).

Knock out of the gene for NPR-B in mice causes severe dwarfism, and the NPR-B knock out mice display seizures, female sterility, and priapism and are therefore not suitable for detailed cardiovascular or neurophysiological phenotyping (Tamura et al., 2004). To overcome this limitation, transgenic rats with ubiquitous overexpression of a dominant-negative NPR-B mutant, lacking the cytoplasmic domain (CMV-NPR-B $\triangle K C$ ) were generated (Langenickel et al., 2006). Receptor homodimerization of NPR-B, leading to a tight contact between the guanylyl cyclase domains, is essential for enzymatic activity. Interestingly, Tamura and Garbers (2003) have described that different NPR-B isoforms resulting from alternative splicing of the primary transcript are present in mouse tissues. In addition to full-length GC-B1, GC-B2 contains a 25 aa deletion in the KHD, and GC-B3 only retains a part of the extracellular ligand-binding domain. When GC-B2 or GC-B3 is expressed coincident with GC-B1, they act as dominant negative isoforms by virtue of blocking formation of active GCB1 homodimers (Tamura and Garbers, 2003). It appears that these splice variants serve as dominant negative regulators of full-length GC-B. Similarly, overexpression of NPR-B $\Delta$ KC leads to functional down-regulation of NPR-B signaling as indicated by blunted CNP-induced cGMP production (Langenickel et al., 2006).

In order to test whether cGMP signaling via neuronal NPR-B affects bidirectional synaptic plasticity in the rat hippocampus, and simultaneously learning and memory, we have generated dominant-negative NPR-B mutant under the control of a neuronspecific promoter (NSE-NPR-B $\triangle \mathrm{KC}$ ) to specifically inhibit NPR$\mathrm{B}$ signaling in the brain. After confirming the expression of the NPR-B $\triangle K C$ mutant and reduction of CNP-dependent cGMP generation in brain membranes we have analyzed bidirectional plasticity in vitro over the range of $1-100 \mathrm{~Hz}$ stimulation in the hippocampal CA1 region. To further evaluate these animals in behavioral experiments we have performed open field, novel object recognition and spatial object recognition (SOR) tests. Our results support the hypothesis that the cGMP-signaling cascade linked to NPR-B and its ligand CNP play an important role in the brain, and in the hippocampus in particular, to modulate bidirectional synaptic plasticity and learning behavior.

\section{MATERIALS AND METHODS GENERATION OF TRANSGENIC RATS WITH SPECIFIC INHIBITION OF NPR-B SIGNALING IN NEURONS}

Transgenic rats overexpressing a dominant-negative NPR-B mutant under the control of a neuron-specific promoter (NSENPR-B $\triangle K C$; Figure 1A) were generated and identified by Southern blotting (Figure 1B) essentially as previously described (Langenickel etal., 2006). After breeding rats to homozygosity, tissue-specific expression of NSE-NPR-B $\triangle K C$ in the brain was verified by reverse transcription (RT)-PCR and Western blotting. RT-PCR, Western blotting and measuring of cGMP levels in brain membrane preparation was performed as previously described (Langenickel et al., 2006; Buttgereit et al., 2010). Primary anti-flag antibodies (Life Technologies, Carlsbad, CA, USA) in combination with secondary anti-rabbit-HRP antibodies were used for detection of NSE-NPR-B $\triangle$ KC-flag in Western blots. For RT-PCR the following primer pairs were used: NSE-NPR-B $\triangle \mathrm{KC}$ forward: GACAGAGAGACTGATTTCGTCC, reverse: TCACTTGTCGTCATCGTCTTTG: GAPDH: forward: CCATGGAGAAGGCTGGGG, reverse: CAAAGTTGTCATGGATGACC. Transgenic rats and control Sprague Dawley rats, aged 6-8 weeks were kept under standard conditions with 12-h lightdark cycle and free access to water and food. The present study was carried out in accordance with the European Communities Council Directive of 24 November 1986 (86/609/EEC) for care of laboratory animals and after approval of the local government ethics committee. All efforts were made to minimize the number of animals used.

\section{SLICE PREPARATION}

Animals were decapitated under deep ether anesthesia. The brain was rapidly taken out of the skull and immersed in ice-cold (1$4^{\circ} \mathrm{C}$ ) sucrose-based solution $\left(87 \mathrm{mM} \mathrm{NaCl}, 25 \mathrm{mM} \mathrm{NaHCO}_{3}\right.$, $10 \mathrm{mM}$ D-glucose, $75 \mathrm{mM}$ sucrose, $2.5 \mathrm{mM} \mathrm{KCl}, 1.25 \mathrm{mM}$ $\mathrm{NaH}_{2} \mathrm{PO}_{4}, 0.5 \mathrm{mM} \mathrm{CaCl}_{2}, 7 \mathrm{mM} \mathrm{MgCl}$, bubbled with $95 \% \mathrm{O}_{2}$ and $\left.5 \% \mathrm{CO}_{2}, \mathrm{pH} 7.4\right)$. Horizontal hippocampal slices (400 $\left.\mu \mathrm{m}\right)$ were cut on a vibratome (Leica VT 1200S). Slices were kept at room temperature. For recordings, the slices were transferred to a submerged recording chamber. During the experiments, the chamber was perfused at a flow rate of $3 \mathrm{ml} / \mathrm{min}$ with oxygenated artificial cerebrospinal fluid (ACSF; $125 \mathrm{mM} \mathrm{NaCl}, 2.5 \mathrm{mM} \mathrm{KCl}$, $1.25 \mathrm{mM} \mathrm{NaH}_{2} \mathrm{PO}_{4}, 25 \mathrm{mM} \mathrm{NaHCO}, 25 \mathrm{mM}$ D-glucose, $2 \mathrm{mM}$ $\mathrm{CaCl}_{2}, 1 \mathrm{mM} \mathrm{MgCl}$, $\mathrm{pH}$ 7.4). All recordings were performed at $32^{\circ} \mathrm{C}$.

\section{RECORDING OF EXTRACELLULAR FIELD POTENTIALS}

Recording electrodes were pulled from borosilicate glass and filled with standard Artificial Cerebrospinal Fluid (ACSF, resistance 1$3 \mathrm{M} \Omega$ ). Extracellular field potentials (FPs) were recorded from 


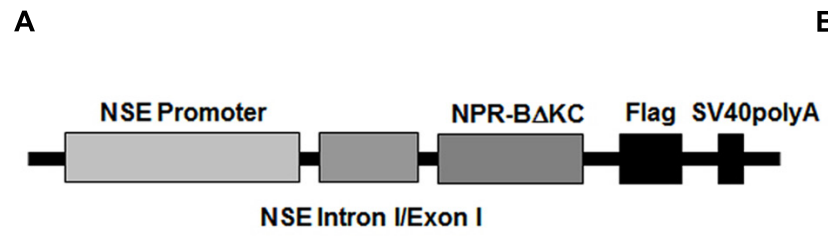

C

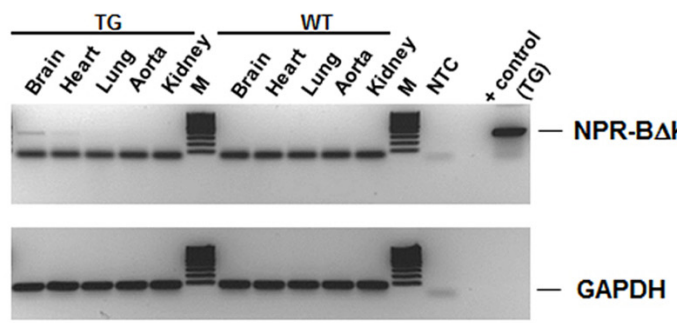

E

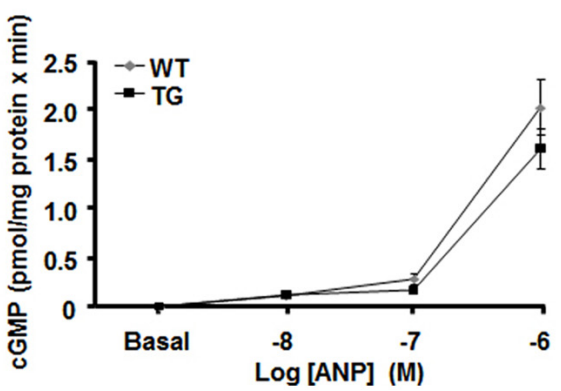

FIGURE 1 | Generation of transgenic rats. (A) Structure of NSE-NPR-B $\triangle K C$ transgenic construct using the neuron-specific enolase promoter (NSE) for neuron-specific expression. The flag/tag was used for immunodetection of the expressed protein. (B) Detection of a founder animal (8809, boxed lane) by Southern blotting. Native NPR-B is present in wild type animals (WT), and native NPR-B and transgene are present in the positive control and founder animal 8809. The transgene construct has a size of $950 \mathrm{bp}$, and native NPR-B of approximately $10 \mathrm{kbp}$. (C) Specific expression of NPR-B $\Delta K C$-Flag was demonstrated by RT-PCR. Negative control (NTC) and positive control

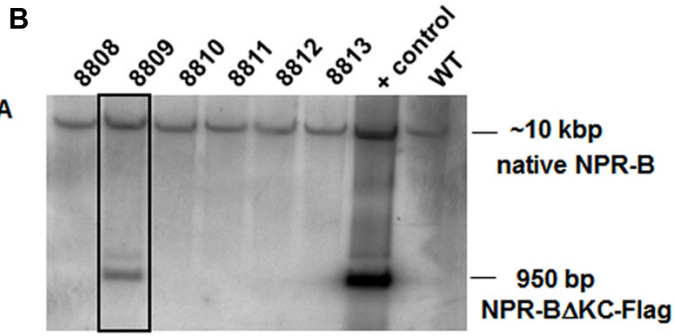

D

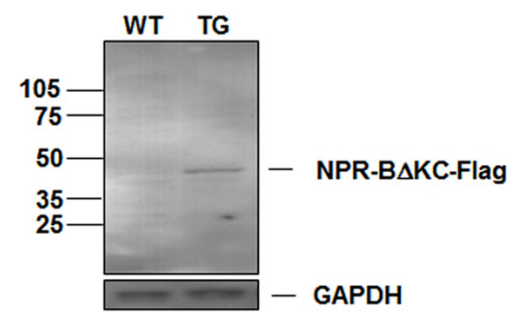

F

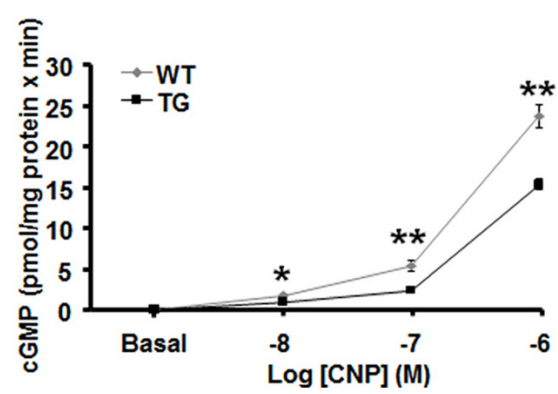

(control + transgene) are shown for the RT-PCR showing NPR-B $\Delta$ KC-Flag expression. PCR for GAPDH expression was used as loading control. M indicates a 100 bp DNA fragment ladder. (D) Specific expression of NPR-B $\triangle$ KC-Flag as shown by Western blotting using an anti-flag antibody using extracts from brain, heart, lung, aorta, and kidney. GAPDH expression is shown as a loading control. (E,F) NPR-B $\triangle K C$ overexpression significantly reduced CNP-dependent, but not ANP-dependent cGMP production in brain membrane preparations in transgene animals (TG) compared to wild-type animals (WT). ${ }^{*} p<0.05,{ }^{*} p<0.01$ vs. wild-type ( $n=6$ per group). stratum radiatum (SR) of area CA1 every $60 \mathrm{~s}$ for at least $10 \mathrm{~min}$ (baseline recording) and then for at least $60 \mathrm{~min}$ following highor low frequency stimulation (HFS or LFS). A concentric bipolar stimulation electrode was placed in SR of area CA1 to stimulate Schaffer collaterals and commissural fibers. FPs were recorded using a MultiClamp 700B amplifier, filtered at $3 \mathrm{kHz}$ and sampled to a computer disk at $10 \mathrm{kHz}$ using the Clampex 10.2 software (Molecular Devices, Sunnyvale, CA, USA). The stimulus intensity was set to evoke $30 \%$ of the maximum FP response in LTP, and $50 \%$ in LTD experiments (50-200 $\mu \mathrm{A}$ with stimulus duration of $100 \mu \mathrm{s})$. The HFS paradigm applied to SR consisted of a $1 \mathrm{~s}$ long stimulus train applied at frequencies of $100,50,30$, or $10 \mathrm{~Hz}$ (pulse duration: $100 \mu \mathrm{s}$, each). For LFS trains 900 pulses were applied at 1 or $5 \mathrm{~Hz}$ (pulse duration: $100 \mu \mathrm{s}$, each; 4-6). FP recordings obtained from SR were analyzed by determining the slope of the ?eld excitatory postsynaptic potential (EPSP) between 10 and $90 \%$ of the peak amplitude. Summary graphs were prepared by normalizing all responses to the baseline obtained during $10 \mathrm{~min}$ prior to HFS or LFS and then averaged across experiments. Only slices with a stable baseline FP response were used for further analysis. All changes in long-term synaptic plasticity were evaluated by averaging responses at 50-60 min post-HFS or LFS and averaging across experiments. For metaplasticity experiments, the frequencies used were HFS $100 \mathrm{~Hz}$ for $1 \mathrm{~s}$ and LFS $1 \mathrm{~Hz}$ for 900 times for $15 \mathrm{~min}$. After the first HFS or LFS for $15 \mathrm{~min}$, LTP was measured for $60 \mathrm{~min}$, then a second HFS or LFS for 15 min was applied, and LTP was measured for another $60 \mathrm{~min}$. CNP (Calbiochem, Merck KGaA, Darmstadt, Germany) was prepared as stock solution, frozen, finally thawed on the day of the experiment and added to ACSF to reach the desired final concentration $(100 \mathrm{nM})$.

\section{BEHAVIORAL TESTS}

The temporal order to test the 11 wild type and the 12 transgenic rats were chosen randomly and changed for each experiment. All trials were recorded by a video camera and exploration time was 
measured by video analysis. The rats' behavior was determined as exploration time when the animal approached closer than $2 \mathrm{~cm}$ to an object with its snout or forepaws (or both). After each trial, the arena and objects were cleaned carefully to avoid the presence of olfactory cues for the next rat. The available objects differed slightly in their shape, size and color. Before the first test - the novel object recognition task - rats were allowed to explore the arena during a habituation trial for $5 \mathrm{~min}$, so that they could adapt to the new environment.

The object recognition task (ORT) was conducted in a squareshaped arena $(100 \mathrm{~cm} \times 100 \mathrm{~cm})$, and used a similar approach as described in a previous report Goh and Manahan-Vaughan (2013). In the training trial (1st trial), two novel objects (i.e., A and B) were presented. Fifty minutes later (second trial) one familiar and one novel object (i.e., A and C) were presented. A further $24 \mathrm{~h}$ later (third trial) object A was presented with a new object (D). The objects were presented in the same location as the objects in the first trial. The objects and the recording chambers were cleaned thoroughly between task trials to ensure the absence of olfactory cues. The objects were distinctly different from one another and heavy so that they could not be moved by the rats.

In the SOR task the objects always remained the same (A and B), and the task was performed as previously described Goh and Manahan-Vaughan (2013). The position of object A remained constant, but in the second and third trial object B was placed in a distinctly new position in the chamber. As in the ORT experiments, the animals were allowed to explore the objects for $5 \mathrm{~min}$. The second and third trials began $50 \mathrm{~min}$ and $24 \mathrm{~h}$ after the first trials, respectively.

In the open field locomotion test we examined motor function by means of measuring spontaneous activity in an open field $(100 \mathrm{~cm} \times 100 \mathrm{~cm})$. Here, we differentiated between the entire running time of the animals and the time spent in the center of the area (without touching the walls).

\section{DATA ANALYSIS}

Data were expressed as mean \pm SEM. Statistical analysis was done with the aid of SigmaStat software (SPSS Inc., Chicago, IL, USA) performing Student's $t$-test (unpaired), Mann-Whitney $U$-test or one-way analysis of variance (ANOVA) with repeated measures. The sample sizes ( $n$ values) for electrophysiological experiments indicate the number of slices from at least three different animals. Differences were considered significant with ${ }^{*} p<0.05$ or $* * p<0.01$.

\section{RESULTS \\ NPR-B-DEPENDENT cGMP SIGNALING IN NSE-NPR-B $\triangle$ KC RATS}

Transgenic rats overexpressing a dominant-negative NPR-B mutant under the control of a neuron-specific promoter (NSENPR-B $\triangle K C$; Figure 1A) were generated and identified by Southern blotting (Figure 1B). After breeding rats to homozygosity, tissue-specific expression of NSE-NPR-B $\Delta K C$ in the brain was verified by RT-PCR. Other tissues, such as heart, lung, aorta and kidney do not express the transgene (Figure 1C). Western blot analysis showed that the NPR-B deletion construct was properly translated into a protein with the expected $\mathrm{Mw}$ of about $45 \mathrm{kDa}$ in the transgenic animals but not in wild type rats (Figure 1D). To test whether expression of the dominant negative NSE-NPR-B $\Delta K C$ mutant leads to the functional down-regulation of the NPR-B signaling, guanylyl cyclase assays in brain membrane preparations were performed (Figures 1E,F). The cGMP production of brain membranes derived from NSE-NPR-B $\Delta$ KC rats stimulated with 10, 100, and $1000 \mathrm{nM}$ ANP was comparable with those derived from wild-type rats, indicating that signaling via NPR-A was not affected in the transgene line (Figure 1E). In contrast, after stimulation with the same doses of CNP cGMP production significantly dampened in brain membrane preparations from NSE-NPR-B $\triangle \mathrm{KC}$ rats compared to wild-type rats. This result confirms the receptor-specific dominant-negative effect of the transgene expression on NPR-B signaling in the brain (Figure 1F).

\section{EFFECT OF NPR-B-DEPENDENT cGMP SIGNALING ON SYNAPTIC PLASTICITY IN HIPPOCAMPAL SLICES IN VITRO}

Here, we investigated the role of NPR-B and cGMP signaling in hippocampal synaptic plasticity. Field extracellular postsynaptic potentials (EPSP) were recorded from the SR of the CA1 by stimulating the Schaffer collaterals using high-frequency stimulation (HFS) at $100 \mathrm{~Hz}$, which typically produces potentiation of the EPSP in the form of LTP. HFS resulted in LTP of $157 \pm 12 \% 1 \mathrm{~h}$ after HFS was applied ( $n=13)$ compared to baseline in wild-type (wt) rats (Figure $2 \mathrm{~A}$ ). In NPR-B $\Delta \mathrm{KC}$ rats, showing reduced CNPdependent cGMP levels in brain membranes, HFS resulted in LTP that in its induction phase was similar to that seen in wt slices but after $60 \mathrm{~min}$ reached $285 \pm 17 \%$ of baseline levels $(n=16$, $p<0.01)$. On the opposite, application of $100 \mathrm{nM}$ CNP to activate hippocampal NPR-B significantly inhibited LTP in wt rats $(115 \pm 10 \%, n=7)$. Since the EPSP recordings in NPR-B $\Delta K C$ rats did not reach saturation levels $60 \mathrm{~min}$ after HFS (Figure 2A), a longer time period of $200 \mathrm{~min}$ was recorded (Figure 2B). EPSP recordings showed a steep rise over the first $30 \mathrm{~min}$, followed by a slower rise over the next $100 \mathrm{~min}$ and finally reached saturation levels at $\sim 250 \%$ of baseline values. This potentiation subsequently decreased somewhat to $\sim 200 \%$ of baseline values $180 \mathrm{~min}$ after HFS (Figure 2B).

\section{THE FREQUENCY-DEPENDENCE OF LTP AND LTD IS MODULATED BY NPR-B ACTIVATION THROUGH CNP AND FUNCTIONAL DOWN-REGULATION OF NPR-B SIGNALING}

Next, we compared the frequency-dependence of synaptic plasticity in wt, homo- and heterozygous NPR-B $\triangle \mathrm{KC}$ animals. The extent of change of synaptic strength was assessed $60 \mathrm{~min}$ after application of afferent stimulation of Schaffer collateral-CA1 synapses (Figure 3 ). In wt animals LTD was elicited at frequencies of 1,5 , and $10 \mathrm{~Hz}$ stimulation. Stimulation at 30,50 , and $100 \mathrm{~Hz}$ led to potentiation of EPSP over baseline. In hetero- and homozygous NPR-B $\triangle \mathrm{KC}$ animals the frequency-dependence of synaptic plasticity was distinctly different to wt controls at frequencies of $5,10,30,50$, and $100 \mathrm{~Hz}(p<0.01, p=0.36$ for $1 \mathrm{~Hz}$ stimulation, Figure 3). Thus, depending on gene dose the NPR-B $\Delta K C$ mutation shifts LTP induction to lower stimulation frequencies compared to wt rats.

We then tested the effect of CNP application (100 nM) on synaptic plasticity in wt slices (Figure 3 ). Here, we observed 

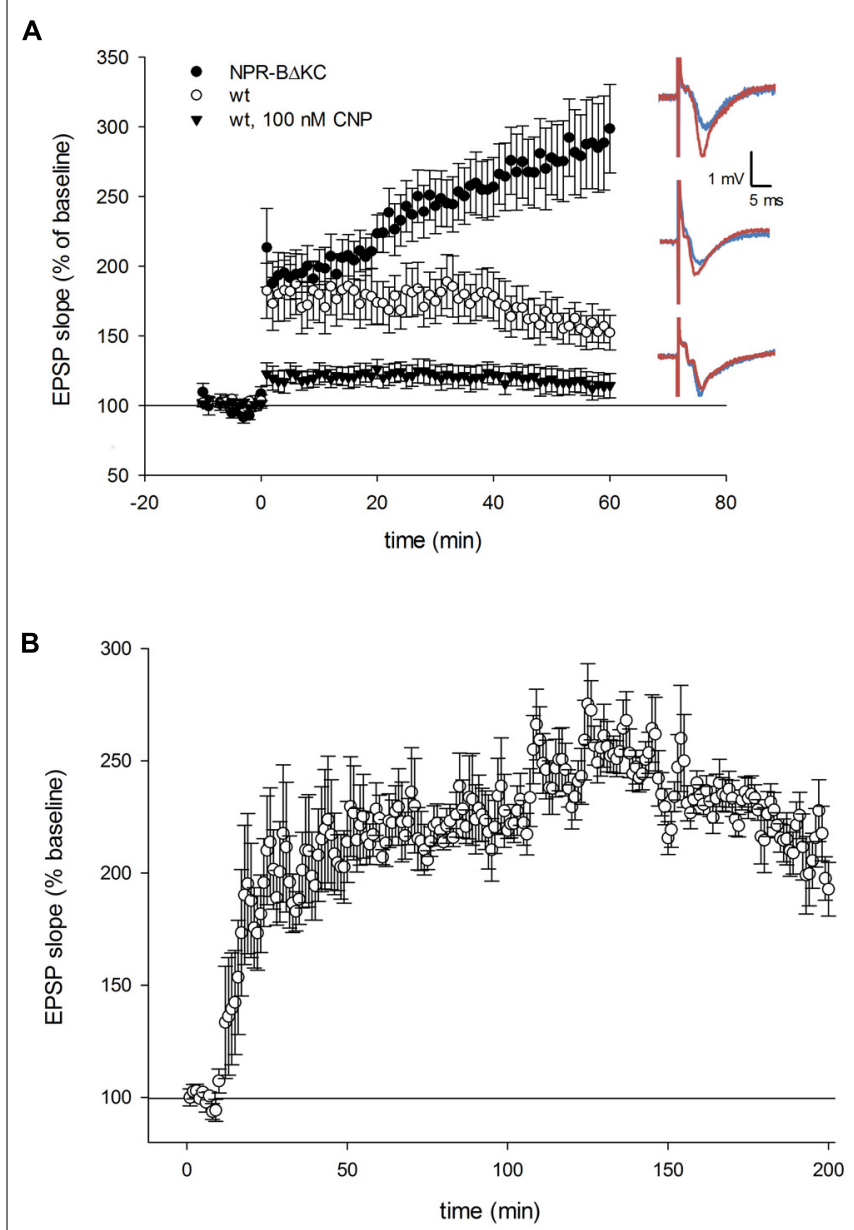

FIGURE 2 | (A) Time course of the relative changes in the excitatory postsynaptic potential (EPSP) slope expressed in \% of baseline stimulation induced by $100 \mathrm{~Hz}$ HFS for $1 \mathrm{~s}$ in hippocampal slices from NPR-B $\Delta$ KC, from wild type rats (wt) and from wild type slices incubated with the NPR-B agonist CNP (100 nM). Sample traces of the electrically evoked EPSPs for the NPR-B $\triangle K C$, wild type slices, and wild type slices incubated with $100 \mathrm{nM}$ CNP are shown on the right, and were recorded $10 \mathrm{~min}$ before and $60 \mathrm{~min}$ following high frequency stimulation (HFS, $100 \mathrm{~Hz}$ for $1 \mathrm{~s}$ ). Note that LTP was more strongly expressed in slices from NPR-B $\triangle K C$ rats (black circles) compared to wt (white circles). Bath application of $100 \mathrm{nM}$ CNP (black triangles) caused attenuation of LTP. (B) The increase of the EPSP slope in NPR-B $\triangle K C$ rats reaches saturation levels at $285 \%$ of baseline stimulation after $120 \mathrm{~min}$. EPSPs decrease slowly to $200 \%$ of baseline stimulation after $180 \mathrm{~min}$.

that although LTD which was elicited using $1 \mathrm{~Hz}$ stimulation was equivalent to that seen in untreated wt controls, LTD induced by $5 \mathrm{~Hz}$ stimulation was significantly enhanced, and potent LTD was elicited by $10 \mathrm{~Hz}$ stimulation. In contrast, CNP-treatment resulted in a failure to induce LTP using $30 \mathrm{~Hz}$ stimulation. Stimulation at 50 and $100 \mathrm{~Hz}$ resulted in only a very small potentiation that was significantly less than that seen in untreated wt controls $(p<0.01)$. Taken together, these data suggest that under conditions of low cGMP levels the threshold for induction of LTP is lowered, whereas when cGMP is strongly elevated via the CNP/NPR-B/cGMP pathway the threshold for LTP induction is raised and LTD induction is facilitated.

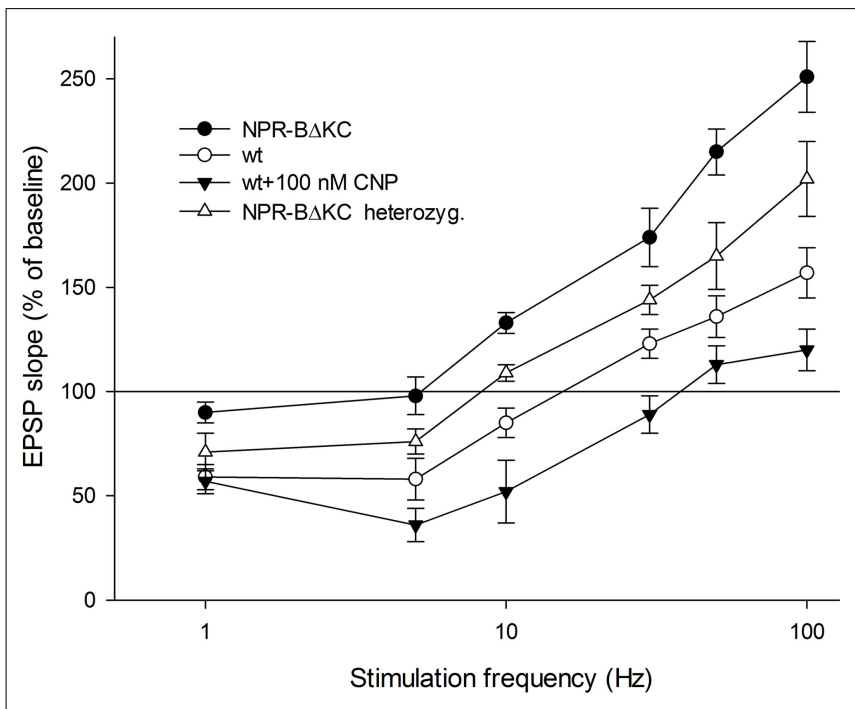

FIGURE 3 | Relative changes in the EPSP slope expressed in \% of baseline stimulation after $\mathrm{HF}$ stimulation at $100 \mathrm{~Hz}$ for $1 \mathrm{~s}$ as recorded 50-60 min after stimulation in hippocampal slices from homozygous NPR-B $\Delta K C$ rats, heterozygous NPR-B $\Delta K C$ rats, slices from wild type rats (wt) and slices from wild type rats incubated with the NPR-B agonist CNP (100 nM). Stimulation frequencies used were 1, 5, 10, 30, 50, and $100 \mathrm{~Hz}$.

\section{POSSIBLE MECHANISMS OF THE EFFECT OF FUNCTIONAL DOWN-REGULATION OF NPR-B SIGNALING ON LTP AND LTD IN HIPPOCAMPAL SLICES IN VITRO}

In search of possible molecular and cellular mechanisms responsible for the observed steady increase in EPSPs over a $60 \mathrm{~min}$ period following HFS, we repeated the experiments in the presence of anisomycin $(20 \mu \mathrm{M})$, an inhibitor of protein synthesis (Figure 4). Anisomycin did not influence the induction of LTP but slightly reduced the potentiation of the EPSP signals in slices from wt rats, and reduced potentiation back to control levels in slices of NPR-B $\triangle \mathrm{KC}$ rats. These results indicate that protein synthesis is involved in the continuous increase of EPSP over a $60 \mathrm{~min}$ period in slices of NPR-B $\triangle K C$ rats. However, the induction of LTP was unaffected by anisomycin in control and NPR-B $\Delta K C$ rats.

We then tested whether NPR-B $\triangle \mathrm{KC}$ affects the intrinsic excitability in hippocampal neurons. Here we measured the EPSP in relation to the stimulation current (Figure 5A). We observed significantly higher responses in slices of NPR-B $\triangle K C$ rats compared to wt using stimulation currents of $0.5-2 \mathrm{~mA}(p<0.05)$. This increase in the input-output relationship indicates positive plastic changes in intrinsic excitability caused by reduced cGMP signaling that may underlie the observed lowering of LTP induction thresholds in NPR-B $\triangle \mathrm{KC}$ rats compared to wt rats.

Moreover, to test whether hippocampal inhibitory activity is affected in NPR-B $\triangle K C$ rats we measured paired-pulse facilitation. Using different interpulse intervals (Figure 5B) we show larger facilitation in slices of NPR-B $\triangle \mathrm{KC}$ rats, particularly at the interpulse interval of 20 and $25 \mathrm{~ms}(p<0.01)$, which is typically indicative of a loss of inhibitory GABAergic activity. 


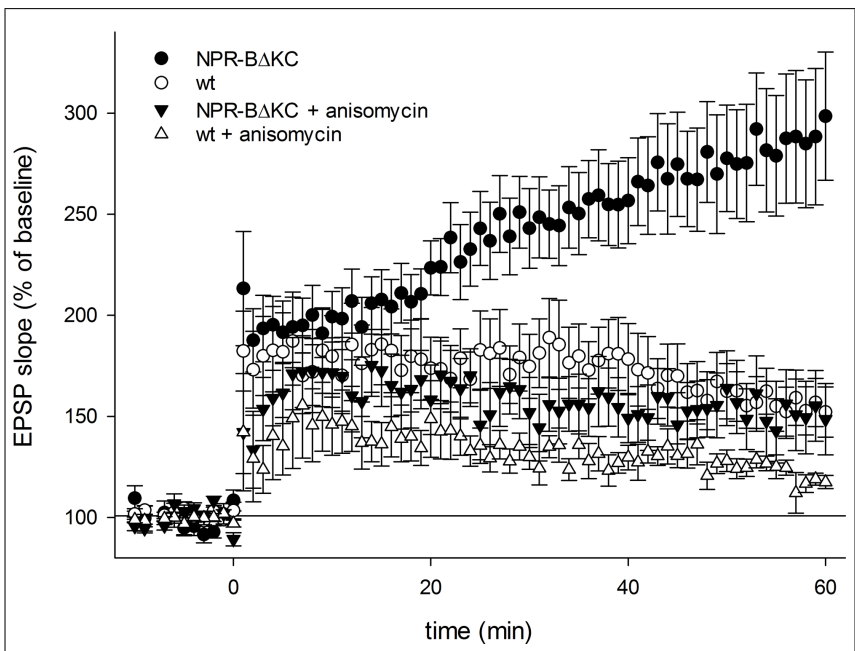

FIGURE 4 | Time course of the relative changes in the EPSP slope expressed in \% of baseline stimulation induced by $100 \mathrm{~Hz}$ HFS for $1 \mathrm{~s}$ in hippocampal slices from NPR-B $\Delta K C$ rats, wild type rats (wt), NPR-B $\Delta K C$ rats incubated with anisomycin $(20 \mu \mathrm{M})$ and wild type slices incubated with anisomycin $(20 \mu \mathrm{M})$.

THE EFFECT OF FUNCTIONAL DOWN-REGULATION OF NPR-B SIGNALING ON METAPLASTICITY IN HIPPOCAMPAL SLICES IN VITRO

Long-term potentiation and LTD depend on the current state of synapses as modulated by intrinsic but also extrinsic influences (Goh and Manahan-Vaughan, 2013). In other words, synaptic plasticity can be modified by activity including previous synaptic inhibition, the activity of modulatory afferents, and hormonal activity, for instance, of natriuretic peptide hormones. This plasticity of synaptic plasticity, also called metaplasticity, may play a role in some mechanisms of memory and learning (Dudek and Bear, 1993). Thus, we assessed effects of NPR$\mathrm{B} \triangle \mathrm{KC}$ on metaplasticity using repetitive HFS $(100 \mathrm{~Hz})$ or LFS $(1 \mathrm{~Hz}) 60 \mathrm{~min}$ after an initial HFS $(100 \mathrm{~Hz})$ or LFS $(1 \mathrm{~Hz}$; Figure 6). Pretreatment with HFS did not increase LTP further following the second HFS in wild type rats, but increased LTP in NPR-B $\triangle$ KC rats. Pretreatment with HFS abolished LTD in response to LFS in wt rats, but further increased LTP in NPR-B $\triangle K C$ rats. Similarly, LFS pretreatment further increased LTP following the second HFS in NPR-B $\Delta K C$ rats, but had no effect in the LFS/LFS combination. Thus, in all combinations of LFS and HFS a significant bigger potentiation was observed after the second stimulation (\% of potentiation $60 \mathrm{~min}$ after first stimulation) in NPR-B $\triangle \mathrm{KC}$ rats compared to $\mathrm{wt}$ rats $(p<0.05)$. These results indicate that the effect of the NPR-B/cGMP signaling system on LTP and LTD also affects metaplasticity.

\section{MEASURING ANXIETY/CURIOSITY AND LEARNING AND MEMORY IN TRANSGENIC RATS EXPRESSING DOMINANT-NEGATIVE NPR-B $\Delta$ KC}

To evaluate changes in learning behavior of NPR-B $\triangle K C$ animals in behavioral experiments, and relate these results with effects of NPR-B $\triangle \mathrm{KC}$ on synaptic plasticity, we performed open field, novel object recognition and SOR tests. In the open field test, anxiety and curiosity were compared between NPR-B $\Delta \mathrm{KC}$ and

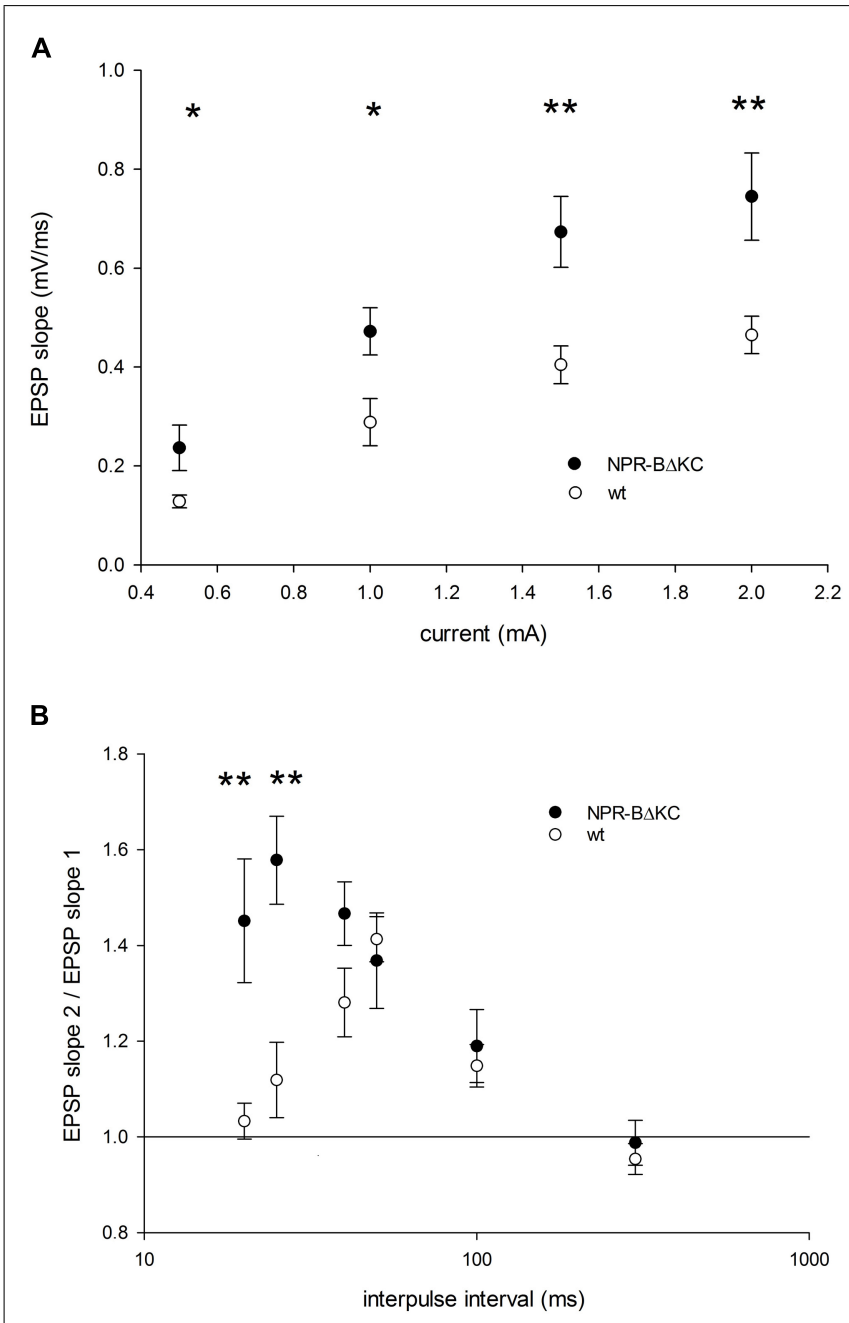

FIGURE 5 | (A) EPSP slope as a function of stimulus current in slices of NPR-B $\triangle K C$ (functional down-regulation of NPR-B signaling) and wild type (wt) rats. EPSP slopes recorded as $\mathrm{mV} / \mathrm{ms}$ were determined between 0.5 and $2 \mathrm{mV}$. Note the greater excitability in hippocampal slices of NPR-B $\Delta K C$ rats with significant differences at $0.5-2 \mathrm{mV}$ stimulus current. (B) Pairedpulse facilitation as a function of interpulse interval current in slices of NPR-B $\triangle K C$ (NPR-B) and wild type (wt) rats. Paired pulses as determined by the ratio of EPSP slope 2/EPSP slope 1 were recorded at 20, 25, 40, 50, 100 , and $300 \mathrm{~ms}$ time intervals. Note the larger facilitation in slices of NPR-B $\triangle K C$ rats at interpulse intervals of 20 and $25 \mathrm{~ms}$. Asterisks indicate significant changes $\left({ }^{*} p<0.05\right.$ and $\left.{ }^{* *} p<0.01\right)$.

control rats. We observed significant differences in locomotion of NPR-B $\triangle K C$ rats in the first trial in the arena (habituation; Figure 7A, $p<0.01$ ). The greater willingness of NPR-B $\Delta \mathrm{KC}$ rats to run can be understood as a sign of greater curiosity and reduced anxiety. However, in the second and third trial after $5 \mathrm{~min}$ and $1 \mathrm{~h}$, respectively, the rats from both groups did not show significant differences in the total distance moved. On the other hand, the NPR-B $\triangle K C$ rats moved significantly longer distances in the central area of the arena compared to the total distance traveled (Figure 7B, $p<0.05$ ). NPR$\mathrm{B} \triangle \mathrm{KC}$ rats also made significantly more visits to the central area (Figure $7 \mathrm{~B}, p<0.01$ ). These results indicate that NPR-B $\Delta \mathrm{KC}$ rats 


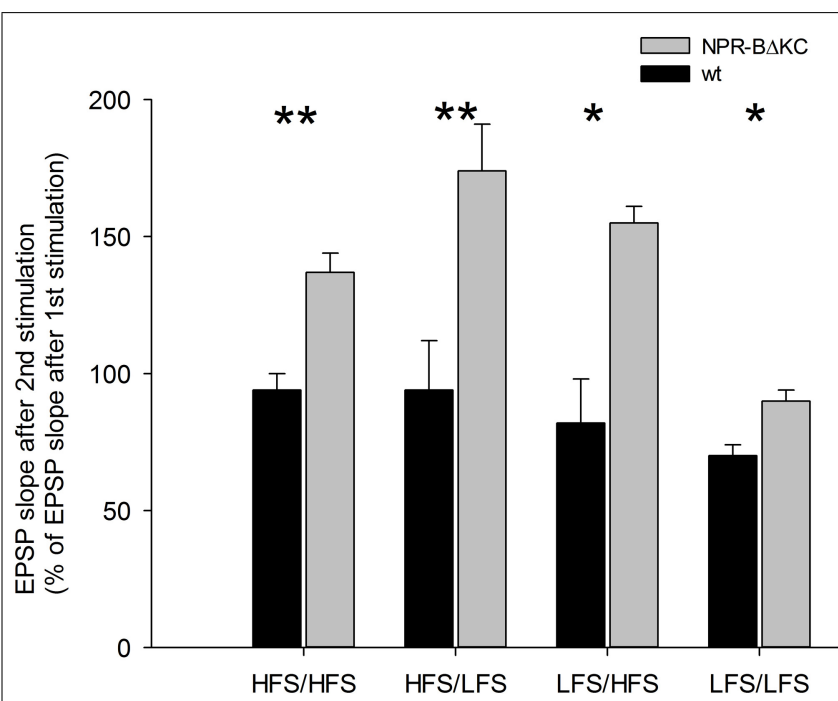

FIGURE 6 | Metaplasticity of EPSP when slices were stimulated a second time with HFS or LFS after an initial HFS, or with HFS or LFS after an initial LFS. LFS, $1 \mathrm{~Hz} ; \mathrm{HFS}, 100 \mathrm{~Hz}$. Bars represent mean values \pm SEM of the relative changes in EPSP slope 50-60 min after the second stimulation in \% of EPSP slope 50-60 min after the first stimulation. Note that LTP is significantly stronger expressed in slices from NPR-B $\triangle K C$ rats in all cases. Asterisks indicate significant changes $\left({ }^{*} p<0.05\right.$ and $\left.{ }^{* *} p<0.01\right)$.

show reduced levels of anxiety leading to enhanced exploratory behavior.

In the novel object recognition test (ORT), upon exposure to the familiar object $(\mathrm{A})$ and the unfamiliar object (C at $50 \mathrm{~min}, \mathrm{D}$ at $24 \mathrm{~h}$ ), one would expect that the animals show a markedly greater interest in the unfamiliar objects compared to object A (22). This was the case both for wt and transgenic animals (Figure 8A). The analysis also revealed a significantly higher exploratory activity for $\mathrm{NPR}-\mathrm{B} \Delta \mathrm{KC}$ rats in the initial $5 \mathrm{~min}$ when objects (A and $\mathrm{B}$ ) were introduced into the arena (Figure 8A, $p=0.065$ ). Object discrimination ratios were calculated to verify that the animals showed a preference of the novel vs. the familiar object (Figure 8B). No significantly different discrimination ratios were recorded in the first trial suggesting that the animals found both novel objects equally interesting. Upon exposure to object A and novel object $\mathrm{C}$ in the second trial, a significant preference for object $C$ over object A was seen in both animal groups. A preference for the novel object was still evident $24 \mathrm{~h}$ after first object exposure, whereby the transgenic animals showed a significantly greater preference for object D compared to wt controls $(p<0.05)$. This indicates that long term memory is facilitated in NPR-B $\triangle \mathrm{KC}$ rats. In the SOR test, the two groups exhibited the expected learning performance, but no significant differences between the two groups were observed (Figure 9A). In the first trial AB1, the second trial after $50 \mathrm{~min}$. and the third trial after $24 \mathrm{~h}$ NPR-B $\Delta \mathrm{KC}$ rats did not exhibited a significantly changed discrimination ratio (Figure 9B). Although NPR-B $\triangle \mathrm{KC}$ rats showed a tendency for an increased discrimination ratio $\mathrm{AB} 3$ in the third trial (Figure 9B), SOR was not significantly different between NPR-B $\triangle K C$ rats and wt animals.

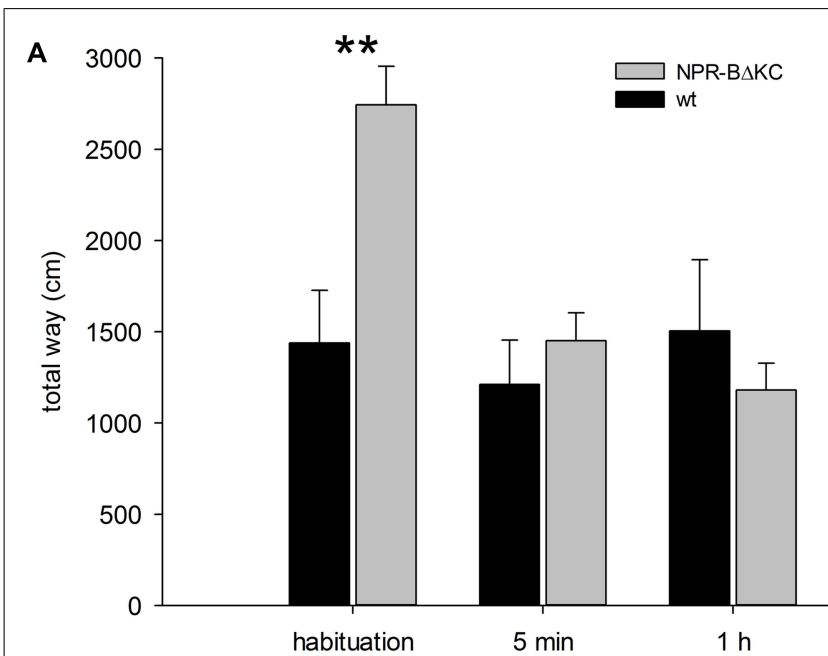

B

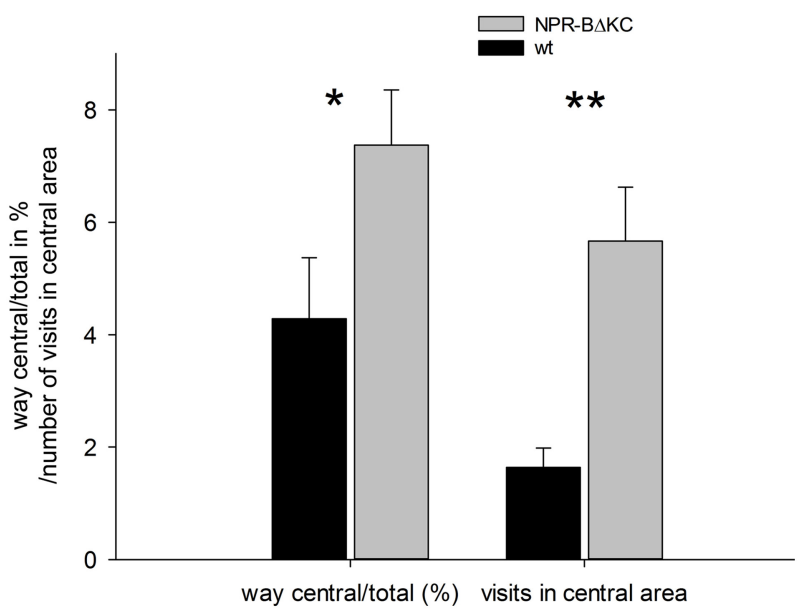

FIGURE 7 | In the open field test NPR-B $\triangle K C$ and wild type rats (wt) were placed in an open field arena and movement of animals was observed to examine exploratory behavior and anxiety. (A) NPR-B $\triangle K C$ rats moved more (total way in $\mathrm{cm}$ ) during initial habituation. In the second trial after $5 \mathrm{~min}$ and the third trial after $1 \mathrm{~h}$ the rats from both groups did not show significant differences in the total distance moved. (B) NPR-B $\Delta K C$ rats showed a higher ratio of movement in the central area versus total movement (way central/total in \%). Transgenic animals also demonstrated an increase in the number of visits in the central area as compared to wt animals. Asterisks indicate significant changes $\left({ }^{*} p<0.05\right.$ and ${ }^{* *} p<0.01$ ).

\section{DISCUSSION}

The second messenger cGMP modulates synaptic transmission and learning processes. This has been attributed mainly to calcium- and nitric oxide (NO)-dependent activation of sGCs (Schuman and Madison, 1991; Arancio etal., 1996; Son et al., 1998). However, other cGMP-producing enzymes including the transmembrane guanylyl cyclase NPR-B are expressed in the brain, including the hippocampal formation. NPR-B is activated by the CNP and produces cGMP in hippocampal neurons (Brackmann et al., 2005). CNP and its receptor NPR-B are strongly expressed in hippocampal regions CA1-3 (Langub etal., 1995; Herman et al., 1996), which are well-described model systems for studying 


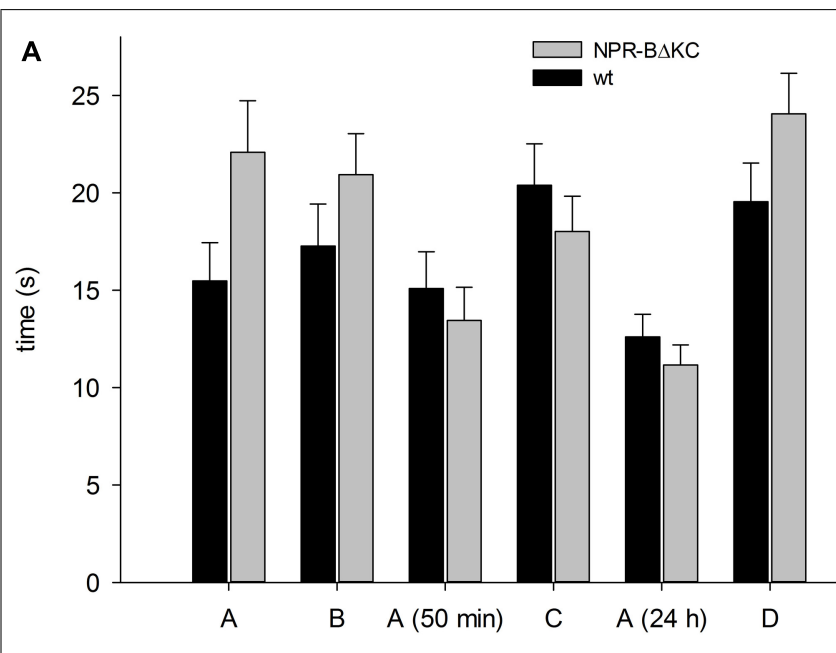

B

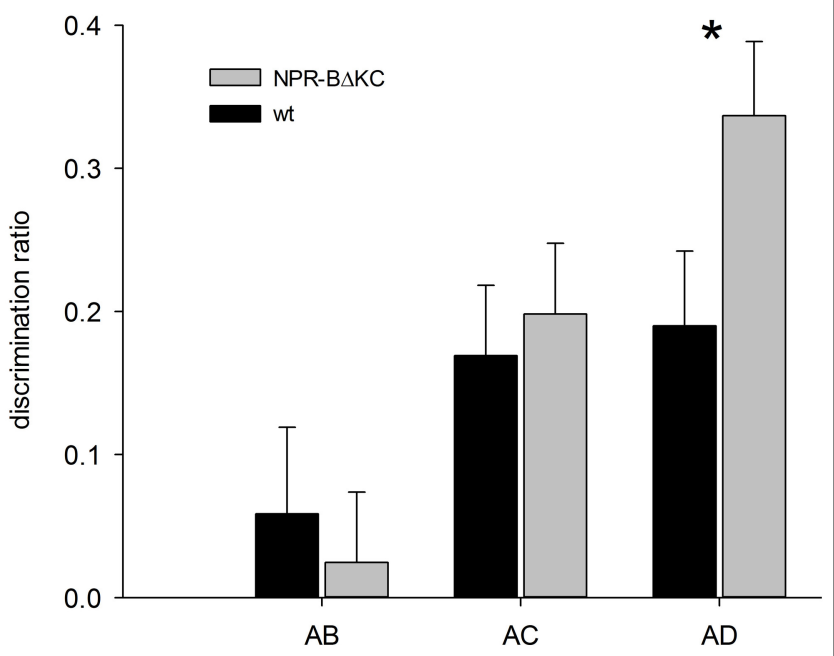

FIGURE 8 | In the novel ORT NPR-B $\triangle K C$ rats and wild type rats (wt) were placed in an open field arena with novel and familiar objects. (A) NPR-B $\triangle K C$ rats revealed higher exploratory activity when different objects ( $A$ and $B$ ) were presented as measured by the time spent in the vicinity to the objects (time in s). Exposure to the familiar (A) or a novel object after $50 \mathrm{~min}(\mathrm{C})$ or $24 \mathrm{~h}$ (D) demonstrated that NPR-B $\Delta K C$ rats had an increased preference for novel object $\mathrm{D}$ after $24 \mathrm{~h}$ compared to wt animals.

(B) Transgenic animals showed a significantly greater preference for the novel object compared to wt controls as analyzed by the discrimination ratio of exploration between new and old object after $24 \mathrm{~h}$ (AD) compared to wt control animals. Asterisks indicate significant changes $\left({ }^{*} p<0.05\right)$.

synaptic plasticity and metaplasticity and their relation to learning and memory (Dudek and Bear, 1993; Manahan-Vaughan and Braunewell, 1999; Braunewell and Manahan-Vaughan, 2001). Evidence that the CNP/NPR-B system is able to modulate synaptic transmission came from our recent study showing that application of CNP, the ligand for NPR-B, negatively affects LTP, but positively affects LTD in hippocampal slices (Decker et al., 2008, 2009, 2010). Our present results indicate that transgenic rats expressing a dominant-negative mutant (NPR-B $\triangle K C$ ) of NPR$\mathrm{B}$ in the brain, display changes of bidirectional plasticity in the opposite way. Rats expressing the NPR-B $\Delta$ KC mutant, which specifically inhibits cGMP production of NPR-B without affecting

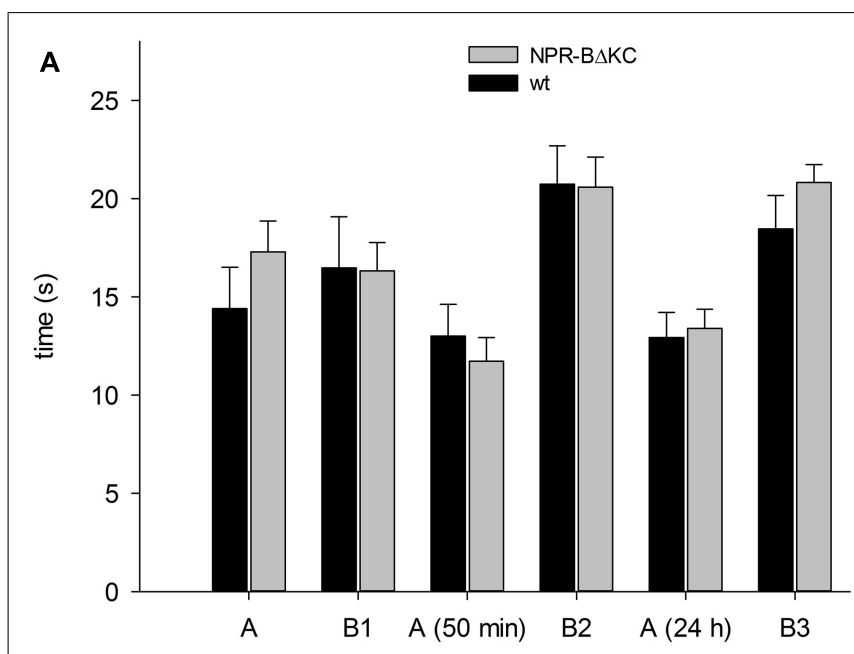

B

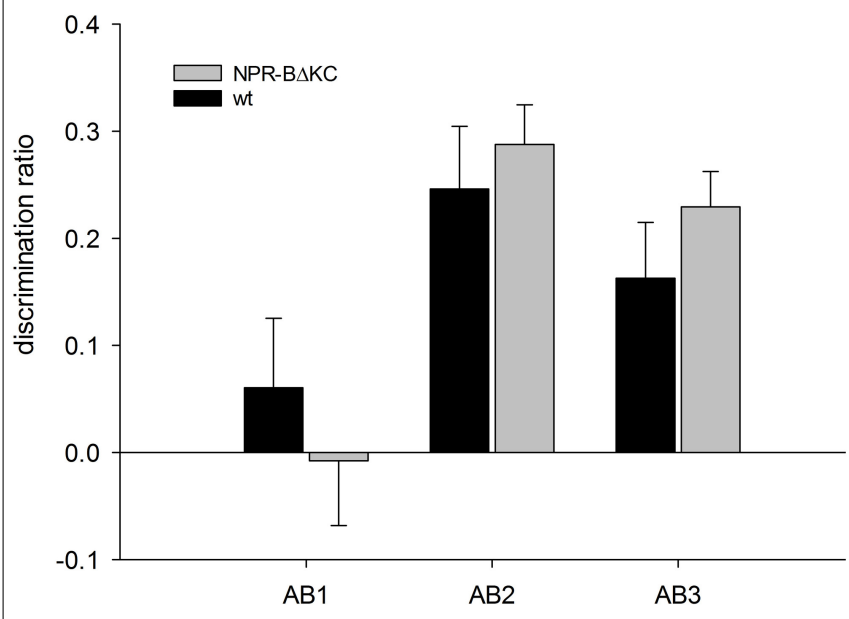

FIGURE 9 | In the spatial object recognition test NPR-B $\triangle K C$ and wild type rats (wt) were placed in an open field arena with two objects ( $A$ and B1, B2, or B3), and with objects B2 and B3 moved to a different place after $50 \mathrm{~min}$ and $24 \mathrm{~h}$, respectively. (A) Both, NPR-B $\triangle K C$ and wild type rats (Wt) showed enhanced interest for the object in a novel place after $50 \mathrm{~min}$ (B2) and $24 \mathrm{~h}$ (B3). (B) The calculation of the discrimination ratio calculation reveals no significant differences in the interest for the object in a novel place after $50 \mathrm{~min}$ (B2) and $24 \mathrm{~h}$ (B3) between NPR-B $\triangle K C$ and wild type rats (wt).

NPR-A, showed enhanced LTP and reduced LTD induction. When frequency-dependence of synaptic modification in the range of 1-100 Hz was assessed transgenic rats expressed LTP at lower stimulation frequencies than wild-type controls and at the same time exhibited enhancements in exploratory and learning behavior. These results indicate that modulation of bidirectional plasticity via NPR-B-dependent cGMP signaling is related to learning and memory behavior in rats, and are in line with the notion that NPR-B and the associated cGMP signaling pathway constitutes a negative feed forward or feedback loop regulating synaptic plasticity.

It is worth mentioning that exploratory activity and spatial learning are associated with LTP and LTD. In rats, exploratory behavior of a new environment containing unfamiliar objects 
and/or familiar objects in new locations facilitated LTD and impaired LTP, whereas exploration of the new environment itself, in the absence of objects, impaired LTD and facilitated LTP (Manahan-Vaughan and Braunewell, 1999; Braunewell and Manahan-Vaughan, 2001; Kemp and Manahan-Vaughan, 2004, 2007, 2008). More recently, it was shown in mice that object recognition facilitates hippocampal LTD and impairs LTP, indicating that both forms of plasticity work in concert in the creation of spatial memory. However, LTP and LTD might contribute to different components of a spatial representation and learning (Goh and Manahan-Vaughan, 2013). In NPR-B $\Delta$ KC mutant rats, showing enhanced LTP and reduced LTD, we found improved object recognition. Although our experiments did not investigate the exact contributions of LTP and LTD to SOR, these results further substantiate the link between LTP and LTD as cellular mechanism required for spatial memory tasks (Goh and Manahan-Vaughan, 2013), and link cGMP signaling via NPR-B with synaptic plasticity and learning.

In regard to the possible molecular and cellular mechanisms of how NPR-B affects synaptic plasticity in the hippocampal network, our results indicate that protein synthesis is involved in the observed effect on EPSPs. The late onset but continuous increase of EPSPs over a prolonged time period in NPR-B $\triangle \mathrm{KC}$ mutant rats was sensitive to the protein synthesis inhibitor anisomycin, indicating that the link between reduced cGMP production and enhanced LTP involves protein synthesis. Moreover, the increase in the input-output relationship of LTP indicates positive alterations in the intrinsic excitability of neurons probably leading to the shift in LTP induction frequencies. This is most likely a sign of loss of inhibitory GABAergic activity in the slices of NPR-B $\triangle$ KC mutant rats. This assumption is in agreement with our previous results showing that CNP activation of NPR-B in hippocampal slices leads to inhibition of LTP by modulating GABA(A)-mediated inhibition of the hippocampal network (Decker et al., 2008, 2009, 2010). Similarly, the effect of the inhibition of NPR-B-dependent cyclase activity on metaplasticity also indicates that there is a loss of negative feedback in the hippocampus of transgenic rats. In our experiments all combinations of LFS and HFS, with the exception of the LFS/LFS combination, led to a significant increase in LTP after the second stimulation in NPR-B $\triangle K C$ rats. These differences in metaplasticity are most likely explained by the fact that $1 \mathrm{~Hz}$ LFS did not induce LTD in the transgenic rats, and that LTP may not have been saturated at $1 \mathrm{~h}$ and typically reached much higher levels than in wt animals. These differences likely contribute to the observed changes in metaplasticity in transgenic animals. Thus, the NPR-B/cGMP signaling system may play a role in metaplasticity, for instance as one of the regulatory feedback mechanism, to link previous experience or the hormonal state of the organism to synaptic plasticity.

In our previous studies we have postulated that the physiological significance of the CNP/NPR-B signaling system in the hippocampus may be to restrict synaptic plasticity and possibly learning and memory behaviors in stress-linked situations and thus link anxiogenic and limbic functions. This assumption was based on preclinical studies in rats and human studies which have shown that natriuretic peptides differentially modulate endocrine and behavioral stress responses (Kellner et al., 2003). CNP enhances the release of ACTH and cortisol (Kellner et al., 2003) and had anxiogenic effects in rats and in humans (Jahn et al., 2001). We have previously shown that exposure of rat strains to stress, in form of a novel open platform environment, induced a significant elevation in serum corticosterone levels but did not facilitate LTD expression (Manahan-Vaughan and Braunewell, 1999). However, different or longer lasting forms of stress activating the NPR-B system are likely to modulate bidirectional plasticity and thus lead to reduced learning and memory-related behavior. Interestingly, in the transgenic rats showing reduction of the NPR-B and cGMP signaling system we observe reduced anxiety levels which are observed as an enhanced exploratory behavior in the open field test as well as in object recognition tests. Enhanced exploratory and novelty seeking behavior is likely linked to learning and memory behavior (Manahan-Vaughan and Braunewell, 1999; Braunewell and Manahan-Vaughan, 2001). Although we have seen significant differences in learning behavior only in novel object recognition and not in the SOR test, enhanced exploratory behavior was noticed in open field and object recognition tests alike. More detailed behavioral analysis will be necessary to further characterize the role of NPR-B for distinct learning and memory paradigms.

Interestingly, GC-C another member of the family of transmembrane guanylyl cyclases (Potter et al., 2009; Potter, 2011), previously thought to be expressed mainly in neurons of the intestine, was identified in midbrain dopaminergic neurons, particularly in midbrain ventral tegmental area and substantia nigra compacta (VTA/SNc) neurons that regulate many important behavioral processes (Gong et al., 2011). Dysfunction of these neurons is associated with attention deficit hyperactivity disorder (ADHD) and schizophrenia. Mice in which GC-C has been knocked out exhibit hyperactivity and attention deficits which resemble behavioral symptoms of ADHD (Gong et al., 2011). Similarly, the functional down regulation of NPR-B in NPR-B $\triangle K C$ rats showed increased activity and exploratory behavior. Thus, dysfunction of receptor guanylyl cyclases and the cGMP-pathway may be involved in a variety of additional CNS disorders, for instance deficits in cGMP signaling pathways and neuroplasticity are hypothesized to underlie the pathophysiology of major depressive disorder (MDD; Reierson et al., 2011). This is in line with the hypothesis that increased cGMP signaling in the brain restricts excitability of neurons, and thereby controls a range of behaviors including hyperactivity, anxiety, attention, learning and memory.

\section{ACKNOWLEDGMENTS}

This work was conducted within the framework of the national priority program (Schwerpunktprogram) SPP1226 Nicotine: molecular and physiological mechanisms in the central nervous system (www.nicotine-research.com) funded by the German Research Foundation (Deutsche Forschungsgemeinschaft, grant DFG $1579 / 8-1$ and 2 to Karl H. Braunewell, MA1843/6 to Denise Manahan-Vaughan). Generation of transgenic rats was supported by DFG grants to Michael Bader and Jens Buttgereit (BA1374/14-1). 


\section{REFERENCES}

Arancio, O., Kiebler, M., Lee, C. J., Lev-Ram, V., Tsien, R. Y., Kandel, E. R., et al. (1996). Nitric oxide acts directly in the presynaptic neuron to produce longterm potentiation in cultured hippocampal neurons. Cell 87, 1025-1035. doi: 10.1016/S0092-8674(00)81797-3

Bartels, C. F., Bukulmez, H., Padayatti, P. R., Ravenswaaijart, C., and Pauli, R. M. (2004). Mutations in the transmembrane natriuretic peptide receptor NPR-B impair skeletal growth and cause acromesomelic dysplisia, type maroteaux. Am. J. Hum. Genet. 75, 27-34. doi: 10.1086/422013

Brackmann, M., Schuchmann, S., Anand, R., and Braunewell, K. H. (2005). Neuronal $\mathrm{Ca} 2+$ sensor protein VILIP-1 affects cGMP signalling of guanylyl cyclase $\mathrm{B}$ by regulating clathrin dependent receptor recycling in hippocampal neurons. J. Cell Sci. 118, 2495-2505. doi: 10.1242/jcs.02376

Braunewell, K. H., and Manahan-Vaughan, D. (2001). Long-term depression: a cellular basis for learning? Rev. Neurosci. 12, 121-140. doi: 10.1515/REVNEURO.2001.12.2.121

Buttgereit, J., Qadri, F., Monti, J., Langenickel, T. H., Dietz, R., Braunewell, K. H., et al. (2010). Visinin-like protein 1 regulates natriuretic peptide receptor B in the heart. Regul. Pept. 161, 51-57. doi: 10.1016/j.regpep.2009. 12.019

Decker, J. M., Wójtowicz, A. M., Bartsch, J. C., Liotta, A., Braunewell, K. H., Heinemann, U., et al. (2010). C-type natriuretic peptide modulates bidirectional plasticity in hippocampal area CA1 in vitro. Neuroscience 169, 8-22. doi: 10.1016/j.neuroscience.2010.04.064

Decker, J. M., Wojtowicz, A., Heinemann, U., and Braunewell, K. H. (2008). C-Type natriuretic peptide modulates pre- and postsynaptic properties in hippocampal area CA1 in vitro. Biochem. Biophys. Res. Commun. 377, 820-825. doi: 10.1016/j.bbrc.2008.10.070

Decker, J. M., Wójtowicz, A. M., Ul Haq, R., Braunewell, K. H., Heinemann, U., and Behrens, C. J. (2009). C-type natriuretic peptide decreases hippocampal network oscillations in adult rats in vitro. Neuroscience 164, 1764-1775. doi: 10.1016/j.neuroscience.2009.09.036

Dudek, S. M., and Bear, M. F. (1993). Bidirectional long-term modification of synaptic effectiveness in the adult and immature hippocampus. J. Neurosci. 13, 2910-2918.

Goh, J. J., and Manahan-Vaughan, D. (2013). Spatial object recognition enables endogenous LTD that curtails LTP in the mouse hippocampus. Cereb. Cortex 23 1118-1125. doi: 10.1093/cercor/bhs089

Gong, R., Ding, C., Hu, J., Lu, Y., Liu, F., Mann, E., et al. (2011). Role for the membrane receptor guanylyl cyclase- $\mathrm{C}$ in attention deficiency and hyperactive behavior. Science 333, 1642-1646. doi: 10.1126/science. 1207675

Herman, J. P., Drucker, D., and Langub, M. C. Jr. (1996). Localization of natriuretic peptide-activated guanylate cyclase mRNAs in the rat brain. J. Comp. Neurol. 369, 165-187. doi: 10.1002/(SICI) 1096-9861(19960527)369:2<165::AIDCNE1>3.0.CO;2-1

Jahn, H. A., Knaudt, K., Ströhle, A., Kiefer, F., Schick, M., and Wiedemann, K. (2001). Alpha-helical-corticotrophin-releasing hormone reverses anxiogenic effects of C-type natriuretic peptide in rats. Brain Res. 893, 21-28. doi: 10.1016/S0006-8993(00)03275-3

Kellner, M., Jahn, H., and Wiedemann, K. (2003). Natriuretic peptides and panic disorder: therapeutic prospects. Expert Rev. Neurother. 3, 381-386. doi: 10.1586/14737175.3.3.381

Kemp, A., and Manahan-Vaughan, D. (2004). Hippocampal long-term depression and long-term potentiation encode different aspects of novelty acquisition. Proc. Natl. Acad. Sci. U.S.A. 101, 8192-8197. doi: 10.1073/pnas.0402650101

Kemp, A., and Manahan-Vaughan, D. (2007). Hippocampal long-term depression: master or minion in declarative memory processes? Trends Neurosci. 30, 111-118. doi: 10.1016/j.tins.2007.01.002

Kemp, A., and Manahan-Vaughan, D. (2008). The hippocampal CA1 region and dentate gyrus differentiate between environmental and spatial feature encoding through long-term depression. Cereb. Cortex 18, 968-977. doi: $10.1093 /$ cercor/bhm136

Kleppisch, T., Wolfsgruber, W., Feil, S., Allmann, R., Wotjak, C. T., Goebbels, S., et al. (2003). Hippocampal cGMP-dependent protein kinase I supports an ageand protein synthesis-dependent component of long-term potentiation but is not essential for spatial reference and contextual memory. J. Neurosci. 23, 6005-6012.
Klyachko, V. A., Ahern, G. P., and Jackson, M. B. (2001). cGMP-mediated facilitation in nerve terminals by enhancement of the spike afterhyperpolarization. Neuron 31, 1015-1025. doi: 10.1016/S0896-6273(01)00449-4

Langenickel, T. H., Buttgereit, J., Pagel-Langenickel, I., Lindner, M., Monti, J., Beuerlein, K., et al. (2006). Cardiac hypertrophy in transgenic rats expressing a dominant-negative mutant of the natriuretic peptide receptor B. Proc. Natl. Acad. Sci. U.S.A. 103, 4735-4740. doi: 10.1073/pnas.0510019103

Langub, M. C. Jr., Watson, R. E. Jr., and Herman, J. P. (1995). Distribution of natriuretic peptide precursor mRNAs in the rat brain. J. Comp. Neurol. 356, 183-199. doi: 10.1002/cne.903560205

Liu, S., Rao, Y., and Daw, N. (2003). Roles of protein kinase A and protein kinase $\mathrm{G}$ in synaptic plasticity in the visual cortex. Cereb. Cortex 13, 864-869. doi: $10.1093 /$ cercor/13.8.864

Manahan-Vaughan, D., and Braunewell, K. H. (1999). Novelty acquisition is associated with induction of hippocampal long-term depression. Proc. Natl. Acad. Sci. U.S.A. 96, 8739-8744. doi: 10.1073/pnas.96.15.8739

Potter, L. R. (2011). Guanylyl cyclase structure, function and regulation. Cell. Signal. 23, 1921-1926. doi: 10.1016/j.cellsig.2011.09.001

Potter, L. R., Yoder, A. R., Flora, D. R., Antos, L. K., and Dickey, D. M. (2009). Natriuretic peptides: their structures, receptors, physiologic functions and therapeutic applications. Handb. Exp. Pharmacol. 191, 341-366. doi: 10.1007/978-3-540-68964-5_15

Reierson, G. W., Guo, S., Mastronardi, C., Licinio, J., and Wong, M. L. (2011). cGMP signaling, phosphodiesterases and major depressive disorder. Curr. Neuropharmacol. 9, 715-727. doi: 10.2174/157015911798376271

Schmidt, H., Stonkute, A., Jüttner, R., Schäffer, S., Buttgereit, J., Feil, R., et al. (2007). The receptor guanylyl cyclase $\mathrm{Npr} 2$ is essential for sensory axon bifurcation within the spinal cord. J. Cell Biol. 179, 331-340. doi: 10.1083/jcb.200707176

Schuman, E. M., and Madison, D. V. (1991). A requirement for the intercellular messenger nitric oxide in long-term potentiation. Science 254, 1503-1506. doi: 10.1126/science. 1720572

Son, H., Yun, F. L., Min, Z., Arancio, O., Kandel, E. R., and Hawkins, R. D. (1998) The specific role of cGMP in hippocampal LTP. Learn. Mem. 5, 231-245.

Tamura, N., Doolittle, L. K., Hammer, R. E., Shelton, J. M., Richardson, J. A., and Garbers, D. L. (2004). Critical roles of the guanylyl cyclase B receptor in endochondral ossification and development of female reproductive organs. Proc. Natl. Acad. Sci. U.S.A. 101, 17300-17305. doi: 10.1073/pnas.0407894101

Tamura, N., and Garbers, D. L. (2003). Regulation of the guanylyl cyclaseB receptor by alternative splicing. J. Biol. Chem. 278, 48880-48889. doi: 10.1074/jbc.M308680200

Telegdy, G., Adamik, A., and Glover, V. (2000). The action of isatin (2,3-dioxoindole) an endogenous indole on brain natriuretic and C-type natriuretic peptideinduced facilitation of memory consolidation in passive-avoidance learning in rats. Brain Res. Bull. 53, 367-370. doi: 10.1016/S0361-9230(00)00359-2

Telegdy, G., Kokavszky, K., and Nyerges, A. (1999). Action of C-type natriuretic peptide (CNP) on passive avoidance learning in rats: involvement of transmitters. Eur. J. Neurosci. 11, 3302-3306. doi: 10.1046/j.1460-9568.1999.00750.x

Conflict of Interest Statement: The authors declare that the research was conducted in the absence of any commercial or financial relationships that could be construed as a potential conflict of interest.

Received: 16 September 2014; accepted: 12 November 2014; published online: 01 December 2014.

Citation: Barmashenko G, Buttgereit J, Herring N, Bader M, Özcelik C, ManahanVaughan D and Braunewell KH (2014) Regulation of hippocampal synaptic plasticity thresholds and changes in exploratory and learning behavior in dominant negative NPR-B mutant rats. Front. Mol. Neurosci. 7:95. doi: 10.3389/fnmol.2014.00095

This article was submitted to the journal Frontiers in Molecular Neuroscience.

Copyright (c) 2014 Barmashenko, Buttgereit, Herring, Bader, Özcelik, ManahanVaughan and Braunewell. This is an open-access article distributed under the terms of the Creative Commons Attribution License (CC BY). The use, distribution or reproduction in other forums is permitted, provided the original author(s) or licensor are credited and that the original publication in this journal is cited, in accordance with accepted academic practice. No use, distribution or reproduction is permitted which does not comply with these terms. 